\title{
Chromatic effects in quadrupole scan emittance measurements
}

\author{
A. Mostacci, ${ }^{1,2}$ M. Bellaveglia, ${ }^{3}$ E. Chiadroni, ${ }^{3}$ A. Cianchi,,${ }^{4,5}$ M. Ferrario, ${ }^{3}$ D. Filippetto,,${ }^{3, *}$ G. Gatti, ${ }^{3}$ and C. Ronsivalle ${ }^{6}$ \\ ${ }^{1}$ Department of Basic and Applied Sciences for Engineering (SBAI), "Sapienza” University of Rome, Rome, Italy \\ ${ }^{2}$ INFN Roma I, Rome, Italy \\ ${ }^{3}$ INFN-LNF, Frascati, Italy \\ ${ }^{4}$ Department of Physics, "Tor Vergata" University of Rome, Rome, Italy \\ ${ }^{5}$ INFN Roma II, Rome, Italy \\ ${ }^{6}$ ENEA C.R., Frascati, Italy
}

(Received 6 April 2012; published 23 August 2012)

\begin{abstract}
A reliable transverse emittance measurement for high-brightness electron beams is of utmost importance for the successful development of fourth generation light sources and for the beam transport in plasmabased accelerators. When the beam exhibits a significant energy spread, typical quadrupole scan emittance measurements may be affected depending on the beam properties and on the quadrupoles arrangement. The emittance degradation induced by chromatic effects in measurements involving magnetic lattices is evaluated analytically for different configurations. Analytical and numerical calculations compared with measurements have been used to evaluate the consequent error on the emittance value measured for single and double quadrupole schemes and for typical operating conditions at the SPARC facility.
\end{abstract}

DOI: 10.1103/PhysRevSTAB.15.082802

PACS numbers: 29.27.Fh, 29.27.Bd, 41.85.Gy

\section{INTRODUCTION AND MOTIVATION}

Fourth generation light sources (e.g. free electron lasers) and advanced particle accelerators (e.g. laser and plasma wakefield accelerators) require the production, acceleration, and transport of high-brightness electron beams. Efforts are done in order to maximize the normalized transverse brightness, $B_{\perp} \propto 2 I_{\text {peak }} / \varepsilon_{n}^{2}$, which means increasing the peak current $I_{\text {peak }}$ and/or minimizing the transverse projected emittance $\varepsilon_{n}$. Increasing the peak current, e.g., reducing the bunch length or increasing the bunch charge, is often done at expenses of transverse emittance. Therefore sources of emittance degradation need to be reduced as much as possible and accurate emittance measurements are mandatory for machine optimization. Under certain conditions, e.g., large transverse beam size, large angular divergence, and large energy spread, it may happen that the emittance measurement itself can vary the emittance, therefore typically overestimating the real emittance of the beam.

The common technique used to measure the transverse emittance of beams not dominated by space charge forces relies on the measurements of the transverse beam size after a linear transport (i.e. linear transformation); the transverse beam size is measured at a given location while changing the upstream optics (quadrupole scan) or it is measured in different locations while keeping the optics fixed (multi-

\footnotetext{
*Present address: LBNL, Berkeley, California 94720-8211, USA.

Published by the American Physical Society under the terms of the Creative Commons Attribution 3.0 License. Further distribution of this work must maintain attribution to the author(s) and the published article's title, journal citation, and DOI.
}

screen measurements) [1]. Interesting studies have been published concerning the impact of several parameters that can affect the measurement including the evaluation of statistics and systematic errors [2,3]; moreover, Refs. [4,5] deal with the effect of the space charge, which is not relevant for the results reported in this manuscript.

In some beam manipulation schemes, such as rf bunch compression (see Ref. [6] and references within) or ps spaced pulse train generation [7], it is foreseen the production and use of beams with energy spread in the few percent range, a value much higher than usual in high-brightness machines. Moreover, beams undergoing ultrahigh gradient acceleration (e.g. wakefield based acceleration) exhibit typically even higher energy spreads, as well as a beam divergence orders of magnitude higher than in conventional rf photoinjectors.

In both cases, the chromatic effect in the quadrupole scan measurement cannot be neglected and the resulting geometric emittance measurement can be affected by systematic errors due to the beam energy spread. On top of that, when the high energy spread (few percent range) is combined to a beam divergence in the few mrad range (e.g. beams in laser-driven plasma wakefield accelerators), the normalized emittance is not simply proportional to the geometric one (details are discussed in Refs. [8,9]). In this paper we will not deal with this aspect, but only with the effect of the energy spread on the geometric emittance measurement, which is the one measured by the quadrupole scan technique.

This paper is motivated by the experience achieved at the SPARC photoinjector [10] where high-brightness longitudinally compressed beams are produced by means of the velocity bunching (VB) $[6,11]$; typically such VB beams exhibit energy spread in the few percent range while the divergence is limited to few hundreds of $\mu \mathrm{rad}$. The SPARC 
scientific program foresees the use of such beams for advanced beam dynamics experiments $[6,12-14]$ and for novel schemes for generation of free electron laser radiation [15].

The SPARC accelerator consists of a 1.6 cells rf gun provided with a solenoid for emittance compensation followed by three SLAC-type traveling wave structures [16]: the $\mathrm{rf}$ compression is accomplished by moving the phase of injection of the beam into the first section (S1) towards the zero crossing. The emittance can be controlled during the compression by focusing the beam with a solenoid embedding S1. The SPARC linac is followed by the high energy $(170 \mathrm{MeV})$ diagnostic section schematically shown in Fig. 1. Single and multiquadrupole scans [1] are performed to determine the transverse emittance by measuring the transverse beam size on the screen $F_{1}$ for different values of current in the quadrupoles $Q_{T} 1, Q_{T} 2$, and $Q_{T} 3$. The line includes also a rf deflector (RFD) used for bunch length and slice emittance measurements or, in combination with the dipole $(D)$, for a complete characterization of the longitudinal phase space on the screen $F_{2}$.

The main advantage in using two quadrupoles with respect to the single quadrupole configuration is the possibility to measure the emittance and Twiss parameters in both planes simultaneously. Moreover, with two quadrupoles there is better control of the beam shape, avoiding losses due to the cutting of beam tails. However, in order to have a beam waist in both planes the needed range of currents is wider and the focusing quadrupole strength stronger, thus the beam undergoes a stronger focalization [17] than in the usual single quadrupole scan. A detailed study of the effects influencing the emittance measurement with the quadrupole scan technique has been done for different lattice configurations, e.g., one and two quadrupoles.

Beams compressed by the VB technique have typically an energy spread of the order of few percent, against a value around $0.1 \%$ when the rf compression is not applied (i.e. the beam is injected on the crest of the S1 rf phase to get the maximum energy at the linac exit). Such large energy spread occurs, in some cases, with large transverse beam size at the entrance of the quadrupole. In this condition, the measured emittance can be substantially different from the real one due to the emittance change induced by chromatic effects in the quadrupoles $[18,19]$. This paper focuses on the effect of chromatism on the emittance measurement of

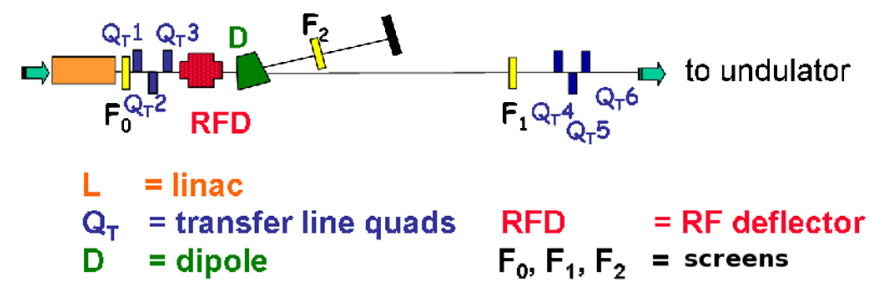

FIG. 1. Layout of SPARC high energy experimental area. Emittance measurements are performed by measuring the transverse beam size at the screen $F_{1}$, which holds a YAG:Ce screen. high-brightness beams, combining analytical formulas with "virtual" and "real" quadrupole scan measurements on typical SPARC beams in different operating conditions.

For reader convenience, the quadrupole scan technique is reviewed in Sec. II, where the measurement uncertainty evaluation is also discussed. A detailed description of the formulas for determining Twiss parameters from the quadrupole scan measurement is presented in the Appendix. Section III shows the emittance dependency on the energy spread in a two-quadrupole (ideal) channel, giving the general formulas used afterwards. Indeed a generic magnetic transport line affects differently particles in the same bunch, depending on their energy. Section IV illustrates the impact on the geometrical emittance of chromatic effects in a single quadrupole. Both cases are considered: correlation and uncorrelation of energy and transverse coordinates (i.e. position $x$ and angle $x^{\prime}$ ). Similar arguments in case of a double quadrupole transport are discussed in Sec. V. Quadrupole-scan virtual measurements are shown to validate analytical calculations in Sec. VI. Measurements on the SPARC beam are presented and discussed in Sec. VII, showing a good agreement with numerical virtual measurements.

\section{THE QUADRUPOLE-SCAN TECHNIQUE TO MEASURE THE EMITTANCE}

Let us assume a generic transport line from some location 0 to another location 1 . The motion of a single particle can then be described in terms of a general transfer matrix [1]

$$
M_{1 \rightarrow 0}=\left(\begin{array}{ll}
A & B \\
C & D
\end{array}\right)
$$

Therefore, the trajectory of the particle in the horizontal trace space $\left(x, x^{\prime}\right)$ [20], from the initial location 0 to a new location 1 , is described through a linear transformation, such as

$$
\left(\begin{array}{l}
x_{1} \\
x_{1}^{\prime}
\end{array}\right)=\left(\begin{array}{ll}
A & B \\
C & D
\end{array}\right)\left(\begin{array}{l}
x_{0} \\
x_{0}^{\prime}
\end{array}\right) \text {. }
$$

Assuming the motion is uncoupled, all the following formulas apply separately both to the vertical and the horizontal plane.

The beam matrix $\Sigma$ can be defined in terms of the second order moments of the distribution in position $\left\langle x^{2}\right\rangle$, angle $\left\langle x^{2}\right\rangle$, and correlation term $\left\langle x^{\prime} x\right\rangle$; thus in the horizontal plane

$$
\Sigma=\left(\begin{array}{cc}
\left\langle x^{2}\right\rangle & \left\langle x x^{\prime}\right\rangle \\
\left\langle x x^{\prime}\right\rangle & \left\langle x^{\prime 2}\right\rangle
\end{array}\right)=\varepsilon\left(\begin{array}{cc}
\beta & -\alpha \\
-\alpha & \gamma
\end{array}\right) .
$$

The optical functions $\beta, \alpha$, and $\gamma$ are thus proportional to the three second order moments of the beam distribution, through the beam emittance. Therefore, the beam size (squared) at location 1 can be expressed in terms of the optical functions and the emittance at an upstream location 0 as

$$
\sigma^{2}=A^{2} \beta \varepsilon-2 A B \alpha \varepsilon+B^{2} \gamma \varepsilon,
$$


where $A$ and $B$ are the elements of the transport line considered [21]. The rms of the distribution, e.g., the horizontal beam size $\sigma=\sqrt{\left\langle x^{2}\right\rangle}$ can be measured at a given position by varying the strength of magnetic lenses (system of quadrupole magnets and/or solenoids) between the locations 0 and 1 .

In a quadrupole scan beam size measurements for at least three different quadrupole settings are required in order to solve for the three independent unknown parameters: $\varepsilon, \beta$, and $\alpha$. Equation (4) can then be written in matrix formalism as

$$
\left(\begin{array}{c}
\sigma_{1}^{2} \\
\sigma_{2}^{2} \\
\ldots \\
\sigma_{N}^{2}
\end{array}\right)=\left(\begin{array}{ccc}
A_{1}^{2} & 2 A_{1} B_{1} & B_{1}^{2} \\
A_{2}^{2} & 2 A_{2} B_{2} & B_{2}^{2} \\
\ldots & \ldots & \ldots \\
A_{N}^{2} & 2 A_{N} B_{N} & B_{N}^{2}
\end{array}\right)\left(\begin{array}{c}
\beta \varepsilon \\
-\alpha \varepsilon \\
\gamma \varepsilon
\end{array}\right),
$$

for a set of $N$ independent $\sigma$ measurement. Such a system is overdetermined and it can be solved by the standard technique of the $\chi^{2}$ minimization, where the difference from the predicted spot size squared [i.e. Eq. (4)] and the measured one $\sigma_{j}^{2}$ is weighted for the uncertainty $u\left(\sigma_{j}^{2}\right)=2 \sigma_{j} u\left(\sigma_{j}\right)$ :

$$
\chi^{2}=\sum_{j=1}^{N}\left[\frac{\sigma_{j}^{2}-A_{j}^{2} \beta \varepsilon+2 A_{j} B_{j} \alpha \varepsilon-B_{j}^{2} \gamma \varepsilon}{2 \sigma_{j} u\left(\sigma_{j}\right)}\right]^{2},
$$

where $\sigma_{j} \pm u\left(\sigma_{j}\right)$ are the rms spot size measurements, while $A_{j}, B_{j}$ are retrieved from the quadrupole line settings. $\chi^{2}$ is minimum when all its derivatives with respect to the coefficients to be determined, i.e. $\beta \varepsilon, \alpha \varepsilon, \gamma \varepsilon$, vanish

$$
\frac{\partial \chi^{2}}{\partial(\alpha \varepsilon)}=0, \quad \frac{\partial \chi^{2}}{\partial(\beta \varepsilon)}=0, \quad \frac{\partial \chi^{2}}{\partial(\gamma \varepsilon)}=0,
$$

which results in a linear system given in Eq. (A1), with the solution being Eq. (A4).

The error on the fit parameters is given by the error or covariance matrix $V$ [22], given for reader convenience in Eq. (A5). The inverse of the covariance matrix is proportional to the second derivative of $\chi^{2}$ with respect to $\beta \varepsilon$, $\alpha \varepsilon, \gamma \varepsilon$, i.e.

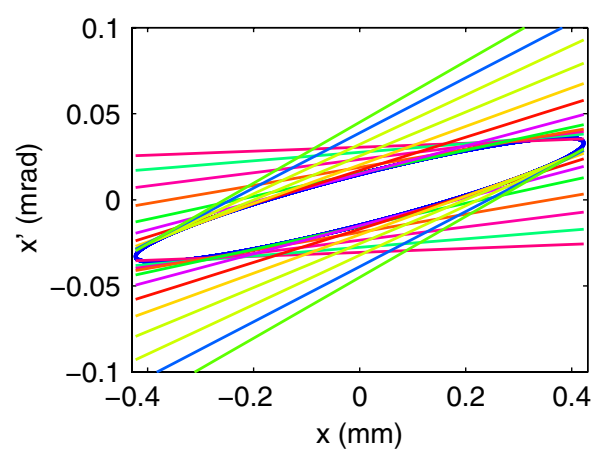

$$
\left(V^{-1}\right)_{i j}=\frac{1}{2} \frac{\partial^{2} \chi^{2}}{\partial a_{i} \partial a_{j}}
$$

with $a_{1}=\beta \varepsilon, a_{2}=-\alpha \varepsilon, a_{3}=\gamma \varepsilon$. The extended expression of $\left(V^{-1}\right)_{i j}$, in terms of the transfer matrix elements $A_{j}$, $B_{j}$, is given in Eq. (A6).

Once the variances and covariances of the fit parameters are known, the errors on the Twiss parameters are the diagonal elements of the $3 \times 3$ matrix defined as $[1,2]$

$$
\left(\nabla_{a} f\right)^{T} V\left(\nabla_{a} f\right)
$$

where

$f=\left(\begin{array}{l}f_{1} \\ f_{2} \\ f_{3}\end{array}\right)=\frac{1}{\sqrt{a_{1} a_{3}-a_{2}^{2}}}\left(\begin{array}{c}a_{1} \\ -a_{2} \\ a_{1} a_{3}-a_{2}^{2}\end{array}\right)=\left(\begin{array}{l}\beta \\ \alpha \\ \varepsilon\end{array}\right)$

and

$$
\nabla_{a} f=\left(\begin{array}{lll}
\frac{\partial f_{1}}{\partial a_{1}} & \frac{\partial f_{2}}{\partial a_{1}} & \frac{\partial f_{3}}{\partial a_{1}} \\
\frac{\partial f_{1}}{\partial a_{2}} & \frac{\partial f_{2}}{\partial a_{2}} & \frac{\partial f_{3}}{\partial a_{2}} \\
\frac{\partial f_{1}}{\partial a_{3}} & \frac{\partial f_{2}}{\partial a_{3}} & \frac{\partial f_{3}}{\partial a_{3}}
\end{array}\right) .
$$

As an example, Fig. 2 shows a typical rms beam sizes measurement at screen $F_{1}$ (see SPARC layout in Fig. 1) in a quadrupole scan, together with the fit of Eq. (4). If the fit has a unique solution the lines enclose only one ellipse, i.e., a unique set of $\beta \varepsilon, \alpha \varepsilon, \gamma \varepsilon$. Each line represents all the points in the transverse trace space, whose transport through the channel would result in $\sigma_{j}$ at the measurement screen.

The consistency of the $\chi^{2}$ fit has been validated by means of a TRACE3D [23] virtual quadrupole scan, where the transverse beam size evolution as a function of the quadrupole gradient has been computed starting from measured emittance and Twiss parameters. As further validation, the rms transverse beam sizes retrieved by TRACE3D have been given as input to the emittance analysis program exhibiting an agreement typically within $2 \%$.

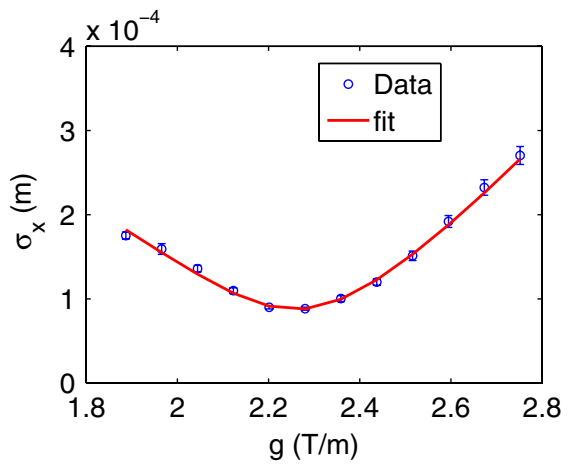

FIG. 2. Left picture: retrieved ellipse in the trace space enclosed by tangent lines corresponding to the set of measurements. Right picture: measured horizontal rms beam size (blue dots) compared to the $\chi^{2}$ fit (red curve) as a function of quadrupole gradient $g$ (measurements in the two-quadrupole configuration; $300 \mathrm{pC}, 147.5 \mathrm{MeV}, \varepsilon_{n}=1.83(0.11) \mathrm{mm} \mathrm{mrad}$ ). 


\section{EMITTANCE CHANGE IN A CHROMATIC TWO-QUADRUPOLE LINE}

In a conventional quadrupole scan, the quadrupole strength is assumed constant for all the particles in the bunch, since energy spread is usually negligible. But if different particles in the same bunch have different energies, the quadrupole kick changes within the bunch itself resulting in a transverse emittance variation. In this section we provide a general formula to account of such an effect, at least for a generic two-quadrupole line; the resulting equation is the basis for all the following discussions.

The quadrupole strength is $k=q g / p$, where $g$ is the field gradient, $p$ is the average momentum, and $q$ is the particle charge. For electrons, in practical units $k\left(m^{-2}\right)=0.2998 *$ $g(T / m) /\left[\beta_{L} \gamma_{L} E_{0}(\mathrm{GeV})\right]$ with $E_{0}$ the particle rest energy, $\gamma_{L}$ the Lorentz factor, and $\beta_{L}$ the ratio between the particle velocity and the speed of light. Since the quadrupole strength depends on the particle energy, the inverse of the focal length $f$ is $1 / f=(k \ell)(1-\delta)=K(1-\delta)$ with $K=k \ell$ and $\ell$ the quadrupole length. $\delta=\Delta p / p$ accounts for the difference of the energy of each particle from the average energy and $\sqrt{\left\langle\delta^{2}\right\rangle}=\sigma_{\gamma}$ is the energy spread. In the thin lens approximation [24], we consider two quadrupoles $(i=1,2)$ separated by a distance $L_{12}$. The transport matrices for each element are

$$
\begin{aligned}
i \text {-quadrupole } & \rightarrow\left(\begin{array}{cc}
1 & 0 \\
-K_{i}(1-\delta) & 1
\end{array}\right) \\
\text { and drift } & \rightarrow\left(\begin{array}{cc}
1 & L_{12} \\
0 & 1
\end{array}\right)
\end{aligned}
$$

and the transfer matrix of the two-quadrupole transport line is

$$
\left(\begin{array}{ll}
A & B \\
C & D
\end{array}\right)=\left(\begin{array}{cc}
1-K_{1} L_{12}(1-\delta) & L_{12} \\
{\left[-\left(K_{1}+K_{2}-K_{1} K_{2} L_{12}\right)+\left(K_{1}+K_{2}-2 K_{1} K_{2} L_{12}\right) \delta+K_{1} K_{2} L_{12} \delta^{2}\right]} & {\left[1-K_{2} L_{12}(1-\delta)\right]}
\end{array}\right)
$$

Being $\varepsilon_{0}, x_{0}, x_{0}^{\prime}$, the emittance, the position, and the divergence just before the first quadrupole, the geometrical emittance after the two quadrupoles is

$$
\begin{gathered}
\varepsilon_{1}^{2}=\left\langle x_{1}^{2}\right\rangle\left\langle x_{1}^{\prime 2}\right\rangle-\left\langle x_{1} x_{1}^{\prime}\right\rangle^{2}, \\
\begin{aligned}
\varepsilon_{1}^{2}= & {\left[\left\langle A^{2}\right\rangle\left\langle C^{2}\right\rangle-\langle A C\rangle^{2}\right]\left\langle x_{0}^{2}\right\rangle^{2}+\left[\left\langle B^{2}\right\rangle\langle\right.} \\
& +\left[\left\langle B^{2}\right\rangle\left\langle C^{2}\right\rangle+\left\langle A^{2}\right\rangle\left\langle D^{2}\right\rangle-2\langle A C\rangle\right. \\
& +\langle B C\rangle)]\left\langle x_{0}^{2}\right\rangle\left\langle x_{0} x_{0}^{\prime}\right\rangle+2\left[\left\langle B^{2}\right\rangle\langle C D\rangle\right.
\end{aligned} \\
\text { Eq. (14) results in } \varepsilon_{1}^{2}=\varepsilon_{0}^{2}, \text { as exp } \\
\text { constant mean energy (i.e. no accele } \\
\text { A. }
\end{gathered}
$$

\section{CHROMATIC EFFECT IN A SINGLE QUADRUPOLE}$$
\varepsilon_{1}^{2}=\left[\left\langle A^{2}\right\rangle\left\langle C^{2}\right\rangle-\langle A C\rangle^{2}\right]\left\langle x_{0}^{2}\right\rangle^{2}+\left[\left\langle B^{2}\right\rangle\left\langle D^{2}\right\rangle-\langle B D\rangle^{2}\right]\left\langle x_{0}^{\prime 2}\right\rangle^{2}+\left[4\langle A B\rangle\langle C D\rangle-(\langle A D\rangle+\langle B C\rangle)^{2}\right]\left\langle x_{0} x_{0}^{\prime}\right\rangle^{2}
$$$$
+\left[\left\langle B^{2}\right\rangle\left\langle C^{2}\right\rangle+\left\langle A^{2}\right\rangle\left\langle D^{2}\right\rangle-2\langle A C\rangle\langle B D\rangle\right]\left\langle x_{0}^{2}\right\rangle\left\langle x_{0}^{\prime 2}\right\rangle+2\left[\left\langle C^{2}\right\rangle\langle A B\rangle+\left\langle A^{2}\right\rangle\langle C D\rangle-\langle A C\rangle(\langle A D\rangle\right.
$$$$
+\langle B C\rangle)]\left\langle x_{0}^{2}\right\rangle\left\langle x_{0} x_{0}^{\prime}\right\rangle+2\left[\left\langle B^{2}\right\rangle\langle C D\rangle+\left\langle D^{2}\right\rangle\langle A B\rangle-\langle B D\rangle(\langle A D\rangle+\langle B C\rangle)\right]\left\langle x_{0}^{\prime 2}\right\rangle\left\langle x_{0} x_{0}^{\prime}\right\rangle .
$$

since $x_{1}, x_{1}^{\prime}$ are the beam position and divergence at the exit of the second quadrupole.

Assuming that the particle energy is uncorrelated from its transverse position/divergence, it results, for instance, $\left\langle A^{2} x_{0}^{2}\right\rangle=\left\langle A^{2}\right\rangle\left\langle x_{0}^{2}\right\rangle$ and so on. Therefore from Eq. (12), one gets
In a drift, Eq. (14) results in $\varepsilon_{1}^{2}=\varepsilon_{0}^{2}$, as expected, for a beam with constant mean energy (i.e. no acceleration). The effect of energy and position/angle correlation is discussed in Sec. IVA.

For a single quadrupole $\left(L_{12}=0, K_{2}=0, K_{1}=K\right)$ the elements of the transfer matrix $M_{1 \rightarrow 0}$ [Eq. (12)] become $A=D=1, B=0$, and $C=-K(1-\delta)$, thus

$\langle C\rangle=-K$ and $\left\langle C^{2}\right\rangle=K^{2}+K^{2}\left\langle\delta^{2}\right\rangle, \quad\langle A C\rangle=K^{2}$,

since $\left\langle x_{0}^{2} \delta\right\rangle=\left\langle x_{0}^{2}\right\rangle\langle\delta\rangle=0$ and $\left\langle x_{0}^{2} \delta^{2}\right\rangle=\left\langle x_{0}^{2}\right\rangle\left\langle\delta^{2}\right\rangle=\sigma_{x}^{2} \sigma_{\gamma}^{2}$ if the energy spread is assumed uncorrelated from the particle transverse position/divergence. Simply applying Eq. (15), most of the terms in Eq. (14) vanish and, reminding that $\sigma_{x}$ is the rms beam size at the quadrupole entrance, the emittance at the exit of the quadrupole reads

$$
\varepsilon_{1}^{2}=\varepsilon_{0}^{2}+K^{2} \sigma_{x}^{4} \sigma_{\gamma}^{2}=\varepsilon_{0}^{2}+\varepsilon_{c}^{2}
$$

where $\varepsilon_{c}^{2}$ accounts for the chromatic effect in the quadrupole; that is,

$$
\varepsilon_{1}=\varepsilon_{0}+\Delta \varepsilon \quad \text { with } \Delta \varepsilon=-\varepsilon_{0}+\sqrt{\varepsilon_{0}^{2}+K^{2} \sigma_{x}^{4} \sigma_{\gamma}^{2}}
$$

giving for very low emittance beams with high energy spread $\left(\varepsilon_{0} \ll \varepsilon_{c}\right)$

$$
\frac{\Delta \varepsilon}{\varepsilon_{0}} \simeq \frac{\varepsilon_{c}}{\varepsilon_{0}}-1+\frac{\varepsilon_{0}}{2 \varepsilon_{c}} \simeq \frac{\varepsilon_{c}}{\varepsilon_{0}},
$$

which corresponds to a linear dependency on quadrupole strength. On the contrary, in the limit of $\varepsilon_{0} \gg \varepsilon_{c}$,

$$
\frac{\Delta \varepsilon}{\varepsilon_{0}} \simeq \frac{\varepsilon_{c}^{2}}{2 \varepsilon_{0}^{2}},
$$

as given in [19], corresponding to a quadratic dependency on quadrupole strength.

In the case of high-brightness electron beams, the normalized beam emittance, $\varepsilon_{n}$, approaches the mm-mrad 
range. The geometric emittance $\left(\varepsilon_{0}=\varepsilon_{n} / \beta_{L} \gamma_{L}\right)$, might be comparable or even smaller than the chromatic term $\varepsilon_{c}$.

\section{A. Effect of energy and position/angle correlation}

The handy formula given in Eq. (16) is reasonably simple and thus very useful for an estimation of the emittance growth due to chromatic effects, but it assumes that correlations between transverse coordinates and energy are negligible. In experimental (real) situations this assumption is not always applicable, especially for highbrightness beams transported in quadrupoles after the interaction with rf fields and solenoids.

In order to include the correlations between energy and transverse position/angles, one can use

$$
x_{1}=x_{0} \quad \text { and } \quad x_{1}^{\prime}=x_{0}^{\prime}+K(1-\delta)
$$

in Eq. (13), thus the emittance after the quadrupole becomes

$$
\begin{aligned}
\varepsilon_{1}^{2}= & \varepsilon_{0}^{2}+\sigma_{x}^{2} K^{2}\left\langle\left(x_{0} \delta\right)^{2}\right\rangle-K^{2}\left\langle x_{0}^{2} \delta\right\rangle^{2} \\
& +2 K\left(\left\langle x_{0} x_{0}^{\prime}\right\rangle\left\langle x_{0}^{2} \delta\right\rangle-\left\langle x_{0} x_{0}^{\prime} \delta\right\rangle \sigma_{x}^{2}\right) .
\end{aligned}
$$

Equation (20) becomes Eq. (16) when the $\left\langle x_{0} \delta\right\rangle=\left\langle x_{0}^{\prime} \delta\right\rangle=0$, i.e., when the particle energy is uncorrelated from its transverse coordinates.

Differently from Eq. (16), that foresees always an increase of emittance due to chromatic effects, Eq. (20) includes also the possibility to get a partial compensation depending on the magnitude and the sign of correlations. This effect has been also highlighted in simulations shown in Sec. VI A.

\section{B. Spot size variation due to non-negligible $\sigma_{\gamma}$ and its effect on the emittance measurement}

Usually in a quadrupole scan the rms spot size $\sigma_{L}$ is measured at a distance $L$ from the quadrupole. The variation of the rms spot size at a distance $L$ from the quadrupole can be computed directly from the quadrupole matrix Eq. (11):

$$
\begin{aligned}
\sigma_{L}^{2}= & \sigma_{L, \delta=0}^{2}+(K L)^{2}\left\langle\delta^{2} x_{0}^{2}\right\rangle+2 K L(1-K L)\left\langle\delta x_{0}^{2}\right\rangle \\
& +2 K L^{2}\left\langle\delta x_{0} x_{0}^{\prime}\right\rangle ;
\end{aligned}
$$

$\sigma_{L, \delta=0}^{2}$ is the spot size at distance $L$ when the quadrupole chromatic effects are negligible, i.e.,

$\sigma_{L, \delta=0}^{2}=(1-K L)^{2}\left\langle x_{0}^{2}\right\rangle+L^{2}\left\langle x_{0}^{\prime 2}\right\rangle+2 L(1-K L)\left\langle x_{0} x_{0}^{\prime}\right\rangle$.

Figure 3 shows the difference between $\sigma_{L}$ and $\sigma_{L, \delta=0}$ for a typical SPARC case. The region around the minimum is critical in the quadrupole scan, and therefore it must not be undersampled in actual measurement. Assuming no correlation between the energy and the transverse position (or divergence), Eq. (21) becomes

$$
\sigma_{L}^{2}=\sigma_{L, \delta=0}^{2}+(K L)^{2} \sigma_{\gamma}^{2} \sigma_{x}^{2}
$$

with $\sigma_{x}$ the spot size at the entrance of the quadrupole and $\sigma_{\gamma}$ the energy spread.

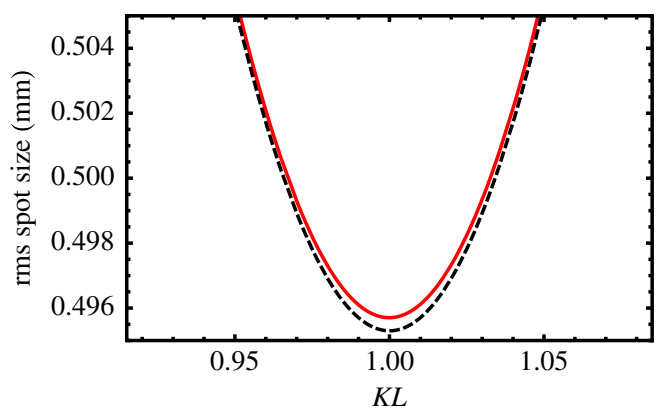

FIG. 3. Variation of the rms spot size on the measurement screen for a single quadrupole scan with (red solid line) and without (black dashed line) energy spread for typical SPARC operations $\left(\sigma_{x}=2 \mathrm{~mm}, \sigma_{x^{\prime}}=100 \mu \mathrm{rad}, \sigma_{x^{\prime} x}=\sigma_{x x^{\prime}}=0\right.$, $L=5 \mathrm{~m}, \sigma_{\gamma}=1 \%$ ).

For a single quadrupole scan, in the thin lens approximation and in absence of correlations between the energy and the transverse position (or divergence), the variation of the spot size squared on the screen at distance $L$ follows a quadratic law in $K L$. An analytical correction to the measured geometric emittance and Twiss parameters due to the energy spread can then be derived as follows. Combining Eqs. (22) and (23), one gets

$$
\sigma_{L}^{2}=(K L)^{2} a+(K L) b+c,
$$

where

$a=\sigma_{x}^{2}\left(1+\sigma_{\gamma}^{2}\right)=\beta \varepsilon\left(1+\sigma_{\gamma}^{2}\right)$,

$b=-2\left[\sigma_{x}^{2}+L\left\langle x x^{\prime}\right\rangle\right]=2 \varepsilon(L \alpha-\beta)$,

$c=\sigma_{x}^{2}+L^{2} \sigma_{x^{\prime}}^{2}+2 L\left\langle x x^{\prime}\right\rangle=\beta \varepsilon-2 L \alpha \varepsilon+L^{2} \gamma \varepsilon$,

with $\sigma_{x}, \alpha, \beta, \gamma, \varepsilon$ the rms spot size, the Twiss parameters, and the emittance at the quadrupole entrance, that is where the Twiss parameter shall be measured. The coefficient $a$ represents the beam size squared at the quadrupole, multiplied by a factor depending on the energy spread, while $c$ is the beam size squared on the screen when the quadrupole is switched off.

When the thin lens approximation is reasonable, Eqs. (24) and (25) are used in actual measurements since the $a, b, c$ coefficients can be retrieved by a second order polynomial fit on the measured $\sigma_{L}^{2}$ values versus $K L$; considering a more precise model of the quadrupole (e.g., thick lenses), one has to use the $\chi^{2}$ fit proposed in Eq. (6). Nevertheless to gain some insight on the physical parameters affecting the systematic errors in the measurements, it makes sense to investigate the thin lens approximation and its analytical solution in more detail.

It is clear that the energy spread does not modify the type of dependence (parabolic in thin lens approximation) of the squared spot size on the $K L$ parameter. The energy spread changes the coefficient of the second order term, modifying the measured emittance value and thus the Twiss parameters. In the following $\alpha, \beta, \varepsilon$ are the optical functions at the entrance of the quadrupole, while $\alpha_{\text {meas }}, \beta_{\text {meas }}, \varepsilon_{\text {meas }}$ are 
the values that we actually measure ( $\varepsilon_{\text {meas }}$ and $\varepsilon$ are geometric emittances). If there were not energy spread, $a=a_{0}=$ $\beta \varepsilon$ (since $\sigma_{\gamma}=0$ ) and

$$
\begin{aligned}
& \varepsilon_{\text {meas }}=\varepsilon=\frac{\sqrt{a_{0} c-b^{2} / 4}}{L} \\
& \beta_{\text {meas }}=\beta=\frac{a_{0}}{\varepsilon} \\
& \alpha_{\text {meas }}=\alpha=\frac{2 a_{0}+b}{2 L \varepsilon} .
\end{aligned}
$$

If $\sigma_{\gamma} \neq 0$, then $a=a_{0}\left(1+\sigma_{\gamma}\right)$ and Eq. (26a) reads

$$
\left(\varepsilon_{\text {meas }} L\right)^{2}=a c-\frac{b^{2}}{4}=(\varepsilon L)^{2}+\sigma_{\gamma}^{2} a_{0} c
$$

that is

$$
\varepsilon_{\text {meas }}^{2}=\varepsilon^{2}+\sigma_{\gamma}^{2} \frac{a_{0} c}{L^{2}}=\varepsilon^{2}+\frac{\sigma_{\gamma}^{2}}{1+\sigma_{\gamma}^{2}} \frac{a c}{L^{2}} .
$$

The error on the measured emittance is the product of three terms: the energy spread, the spot size at the lens $\left(a_{0}\right)$, and the spot size on the screen with quadrupole off $(c)$, over the drift length $L$ squared. The rightmost term in the equality of Eq. (28) can be actually used to compute the systematic error to the measured emittance, since $a, c$ can be retrieved by the fitting of the data [according to Eq. (24)] and $\sigma_{\gamma}$ is usually measured independently.

In the limit of $\sigma_{x}<1 \mathrm{~mm}, \sigma_{x^{\prime}}>1 \mathrm{mrad}, L$ greater than few meters and no correlations between position and angle $\left(\left\langle x x^{\prime}\right\rangle=\alpha=0\right)$, Eq. (28) can be further simplified, since $a_{0}=\sigma_{x}^{2}, c / L^{2} \approx \sigma_{x^{\prime}}^{2}$, and

$$
\varepsilon_{\text {meas }}^{2} \approx \varepsilon^{2}+\sigma_{\gamma}^{2} \sigma_{x}^{2} \sigma_{x^{\prime}}^{2}
$$

Therefore the bigger $\sigma_{x}$ and/or $\sigma_{x^{\prime}}$ are at the quadrupole entrance, the more the energy spread affects the emittance measurement. Such simplification is not always applicable at SPARC, but it may be interesting in plasma wakefield accelerated beams which, due to the extremely intense focusing forces inside the plasma, exhibit typically divergence larger than those typical of beams from conventional accelerators [8].

The Twiss parameters can be computed as well, analogously to Eqs. (26b) and (26c), that is

$$
\begin{aligned}
& \beta_{\text {meas }}=\beta \frac{1+\sigma_{\gamma}^{2}}{\varepsilon_{\text {meas }} / \varepsilon}, \\
& \alpha_{\text {meas }}=\frac{1}{\varepsilon_{\text {meas }} / \varepsilon}\left(\alpha+\frac{\beta}{L} \sigma_{\gamma}^{2}\right),
\end{aligned}
$$

showing that the measurements tend to underestimate the $\beta$ values. In absence of correlations between energy and transverse coordinates and in the limit of validity of thin lens approximation, the above relations can be directly used to correct the emittance and Twiss parameters also in the presence of energy spread, once its measurement is provided.

In the presence of correlations an analytical expression of the error on measured emittance and Twiss parameters, induced by chromatics effects, can be also retrieved following the same procedure, but starting from the more general Eq. (21). However, in this case also the coefficient of the term proportional to $K L$ [in Eq. (24)] is modified by the energy spread. Therefore, if the correlation terms are unknown, the correction based only on the measurement of the energy spread cannot be directly applied as before.

\section{CHROMATIC EFFECT IN THE DOUBLE QUADRUPOLE}

Let us consider the case of two quadrupoles at a distance $L_{12}$ and strength depending on the particle energy, so that $K_{i}(1-\delta)$ for $i=1,2$. For symmetry reasons $\langle\delta\rangle=0,\left\langle\delta^{3}\right\rangle=0$, thus neglecting the higher order terms $\left\langle\delta^{2}\right\rangle^{2},\left\langle\delta^{4}\right\rangle,\left\langle\delta^{2}\right\rangle^{4}, \ldots$, using in Eq. (14) the transfer matrix defined in Eq. (12), one gets

$$
\begin{aligned}
\varepsilon_{1}^{2}= & \varepsilon_{0}^{2}+f\left(K_{1}, K_{2}, L_{12}\right) \sigma_{\gamma}^{2} \sigma_{x}^{4} \\
& +g\left(K_{1}, K_{2}, L_{12}\right) \sigma_{\gamma}^{2} \sigma_{x^{\prime}}^{4}+h\left(K_{1}, K_{2}, L_{12}\right) \sigma_{\gamma}^{2} \sigma_{x x^{\prime}}^{2} \\
& +t\left(K_{1}, K_{2}, L_{12}\right) \sigma_{\gamma}^{2} \sigma_{x}^{2} \sigma_{x^{\prime}}^{2} u\left(K_{1}, K_{2}, L_{12}\right) \sigma_{\gamma}^{2} \sigma_{x}^{2} \sigma_{x x^{\prime}} \\
& +v\left(K_{1}, K_{2}, L_{12}\right) \sigma_{\gamma}^{2} \sigma_{x^{\prime}}^{2} \sigma_{x x^{\prime}},
\end{aligned}
$$

where $\sigma_{\gamma}^{2}=\left\langle\delta^{2}\right\rangle, \sigma_{x}^{2}=\left\langle x_{0}^{2}\right\rangle, \sigma_{x^{\prime}}^{2}=\left\langle x_{0}^{\prime 2}\right\rangle, \sigma_{x x^{\prime}}=\left\langle x_{0} x_{0}^{\prime}\right\rangle$ at the first quadrupole entrance, and

$$
\begin{aligned}
f\left(K_{1}, K_{2}, L_{12}\right)= & \left\{K_{2}+K_{1}\left[1+K_{2} L_{12}\left(-2+K_{1} L_{12}\right)\right]\right\}^{2} \\
g\left(K_{1}, K_{2}, L_{12}\right)= & L_{12}^{4} K_{2}^{2} \\
h\left(K_{1}, K_{2}, L_{12}\right)= & 4 L_{12}\left(1-K_{1} L_{12}\right)\left(2 K_{1} K_{2} L_{12}\right. \\
& \left.+K_{2}^{2} L_{12}-3 K_{1} K_{2}^{2} L_{12}^{2}\right) \\
t\left(K_{1}, K_{2}, L_{12}\right)= & 2 K_{2}\left(K_{2}-K_{1}\right) L_{12}^{2}-4 K_{1} K_{2}^{2} L_{12}^{3} \\
& +2 K_{1}^{2} K_{2}^{2} L_{12}^{4} \\
u\left(K_{1}, K_{2}, L_{12}\right)= & 4 K_{2} L_{12}\left(1-K_{1} L_{12}\right)\left\{K_{2}\right. \\
& \left.+K_{1}\left[1-K_{2} L_{12}\left(2-K_{1} L_{12}\right)\right]\right\} \\
v\left(K_{1}, K_{2}, L_{12}\right)= & 4 K_{2}^{2} L_{12}^{3}\left(1-K_{1} L_{12}\right)
\end{aligned}
$$

The previous Eqs. (31) and (32) agree with known limits. If only the first quadrupole is powered, i.e. $K_{2}=0$, then

$$
\left.\varepsilon_{1}^{2}\right|_{K_{2}=0}=\varepsilon_{0}^{2}+K_{1}^{2} \sigma_{x}^{4} \sigma_{\gamma}^{2}
$$

which is exactly Eq. (16).

Equation (31) shows that the chromatic contribution to the emittance after a doublet is smaller if the first quadrupole is converging; indeed $(\tilde{K}>0)$

$$
\begin{aligned}
& \left.\varepsilon_{1}^{2}\right|_{K_{1}=\tilde{K}, K_{2}=-\tilde{K}}-\left.\varepsilon_{1}^{2}\right|_{K_{1}=-\tilde{K}, K_{2}=\tilde{K}} \\
& \quad=-8 \tilde{K}^{3} L_{12}^{2} \sigma_{\gamma}^{2}\left(k^{2} L_{12} \sigma_{x}^{2}+L_{12} \sigma_{x^{\prime}}^{2}+2 \sigma_{x x^{\prime}}\right)\left(\sigma_{x}^{2}+L_{12} \sigma_{x x^{\prime}}\right),
\end{aligned}
$$

that is, assuming that the increase due to $\sigma_{x}^{4}$ is dominant,

$$
\left.\varepsilon_{1}^{2}\right|_{K_{1}=\tilde{K}, K_{2}=-\tilde{K}}-\left.\varepsilon_{1}^{2}\right|_{K_{1}=-\tilde{K}, K_{2}=\tilde{K}} \simeq-8 \tilde{K}^{5} L_{12}^{3} \sigma_{x}^{4} \sigma_{\gamma}^{2} .
$$




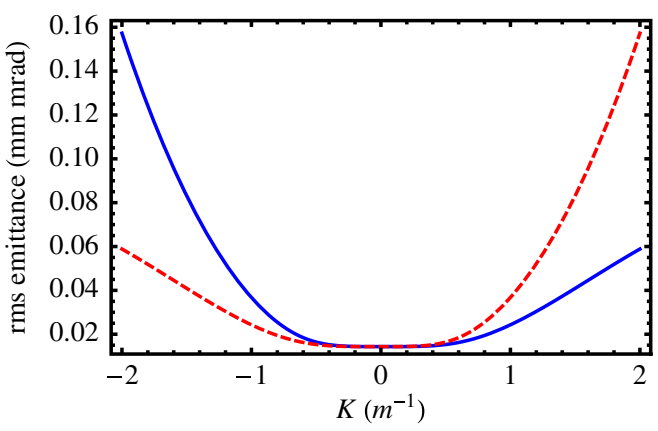

FIG. 4. Variation of the rms geometric emittance in a doublet: the first quadrupole is converging (diverging) in the blue solid (red dashed) line $\left(\sigma_{x}=1.7 \mathrm{~mm}, \sigma_{x^{\prime}}=76 \mu \mathrm{rad}, \sigma_{x^{\prime} x}=\sigma_{x x^{\prime}}=\right.$ $0.1284 \mathrm{~mm} \operatorname{mrad}, L_{12}=0.45 \mathrm{~m}, \sigma_{\gamma}=1 \%$ ).

This asymmetry can affect double quadrupole scan for large beam spot size. Figure 4 shows the emittance variation at the exit of a doublet as a function of the quadrupole strength $K$; for example, when $K>0$ the blue line refers to the first quadrupole focusing (i.e. $K_{1}=-K_{2}=K$ ) while the red dashed line concerns the first quadrupole defocusing $\left(-K_{1}=K_{2}=K\right)$. The beam parameters refer to a SPARC operation condition (see the second column of Table I in Sec. VIA). The chromatic emittance variation is smaller when the first quadrupole is focusing as shown from the blue (red) line for $K>0(K<0)$, as shown by Eqs. (34) and (35). Therefore for a given doublet quadrupole scan, horizontal and vertical emittance are affected differently if the only positive (or negative) $K$ are scanned, as occurring in a typical double quad-scan measurement.

\section{Spot size variation due to non-negligible $\boldsymbol{\sigma}_{\gamma}$}

Assuming that the distance between the two quadrupoles is $L_{12}$ and $L$ is the distance between the last quadrupole and the measurement screen,

$$
\begin{aligned}
\sigma_{L}^{2}= & \sigma_{L, \delta=0}^{2}+\left\{K_{1}^{2} L_{12}^{2}+2 K_{1} L_{12}\left(K_{1}+2 K_{2}-3 K_{1} K_{2} L_{12}\right) L\right. \\
& +\left[\left(K_{1}+K_{2}\right)^{2}-6 K_{1} K_{2}\left(K_{1}+K_{2}\right) L_{12}\right. \\
& \left.\left.+6 K_{1}^{2} K_{2}^{2} L_{12}^{2}\right] L^{2}\right\} \sigma_{\gamma}^{2} \sigma_{x}^{2}+\left(K_{2} L_{12} L\right)^{2} \sigma_{\gamma}^{2} \sigma_{x^{\prime}}^{2} \\
& +\left(2 K_{2} L_{12} L\left\{K_{2} L+K_{1}\left[2 L+L_{12}\left(2-3 K_{2} L\right)\right]\right\}\right) \sigma_{\gamma}^{2} \sigma_{x x^{\prime}},
\end{aligned}
$$

with

$$
\begin{aligned}
\sigma_{L, \delta=0}^{2}= & \left\langle\left\{ x-L_{12}\left(-1+K_{2} L\right)\left(x^{\prime}-K_{1} x\right)\right.\right. \\
& \left.\left.+L\left(x^{\prime}-\left[K_{1}+K_{2}\right) x\right]\right\}^{2}\right\rangle,
\end{aligned}
$$

that is

$$
\begin{aligned}
\sigma_{L, \delta=0}^{2}= & 2\left[L+L_{12}\left(1-K_{2} L\right)\right]\left\{1-K_{2} L-K_{1}\right. \\
& \left.\times\left[L+L_{12}\left(1-K_{2} L\right)\right]\right\} \sigma_{x x^{\prime}}^{2} \\
& ++\left\{1-K_{2} L-K_{1}\left[L+L_{12}\left(1-K_{2} L\right)\right]\right\}^{2} \sigma_{x}^{2} \\
& +\left(L_{12}+L-K_{2} L_{12} L\right)^{2} \sigma_{x^{\prime}}^{2} .
\end{aligned}
$$

In case of a symmetric doublet, i.e. $K_{1}=-K_{2}=K>0$, the variation of the rms spot size as function of $K L$ is indicated in Fig. 5 for the horizontal (left) and vertical (right) plane. The difference in spot size with (red solid line) and without (black dashed line) energy spread is greater with respect to the single quadrupole scan (compare with Fig. 3). This difference is even greater in case of two-quadrupole scan (Fig. 5) in the plane where the first quadrupole is defocusing (Fig. 5, right plot). Figures 3 and 5 use the same beam parameters and the same span in the vertical and the horizontal axis allowing the reader to compare directly the difference between the single and doublet quadrupole scan. An equation analogous to Eq. (24) could also be written in the case of symmetric doublet but the $\sigma_{L}^{2}$ will depend now on the fourth power of $K L$. Therefore the derivation of the systematic error to the emittance measurement [i.e. a relation analogous to Eq. (28)] is outside the aim of this manuscript.

\section{VIRTUAL MEASUREMENTS}

This section compares virtual quadrupole scan measurements for the SPARC layout using a single quadrupole and the doublet for the same beam. The results validate the analytical calculations presented above and explain the difference in the measured emittance encountered in some conditions at SPARC [e.g. if the quadrupole scan is done with a doublet with the first quadrupole focusing in the horizontal plane and defocusing in the vertical plane $\left(K_{1}=-K_{2}=K>0\right)$ or vice versa $\left.\left(K_{2}=-K_{1}=K>0\right)\right]$. Start-to-end simulations of the beam evolution for typical SPARC operations are done with the macroparticle TSTEP [25] code by using 50000 macroparticles. The average beam parameters (i.e. emittance, second order moments, correlations) at the linac exit are computed on the simulation results. Three virtual measurements in three different beam configurations (Table I) meaningful for the actual SPARC operation have been done, by propagating a $300 \mathrm{pC}$ beam in the diagnostic beam line of Fig. 1 up to the measurement screen (i.e. screen $F_{1}$ of Fig. 1) and analyzing the virtual scan data according to Sec. II. It has been also verified, by switching on and off the space charge routine in TSTEP, that the beam spot evolution is not affected by space charge forces; therefore from this point of view the quadrupole scan technique can be applied. For each configuration, different magnetic lattices for the quadrupole scan have been explored, i.e., single quadrupole for both horizontal and vertical planes, two quadrupoles with the first one focusing $\left(Q_{T} 1\right.$ of Fig. 1) and the second one defocusing ( $Q_{T} 3$ of Fig. 1) in the horizontal plane, and vice versa. The emittance variation induced by chromatic effects during the scan has also been recorded. Table I shows the operating condition and the main beam parameters at the linac exit (i.e. screen $F_{0}$ of Fig. 1) for three cases differing in the chromatic contribution. The energy is lower in Case 2 and Case 3 (rf compression on) 
TABLE I. Operating conditions and computed beam parameters in the three configurations used for the virtual measurements. $\sigma_{x, F_{0}}, \sigma_{y, F_{0}}$ are the beam rms spot size at the linac exit (screen $F_{0}$ of Fig. 1) while $\varepsilon_{x, \text { un }}, \varepsilon_{y, \text { un }}$ are the transverse geometrical emittances.

\begin{tabular}{lccc}
\hline \hline & Case 1 & Case 2 & Case 3 \\
\hline Rf compression & Off & On & On \\
Solenoid on S1 & Off & Off & On \\
Energy $(\mathrm{MeV})$ & 148 & 105 & 105 \\
$\sigma_{x, F_{0}}(\mathrm{~mm})$ & 0.571 & 1.735 & 0.343 \\
$\sigma_{y, F_{0}}(\mathrm{~mm})$ & 0.595 & 1.788 & 0.341 \\
$\sigma_{\gamma}(\%)$ & 0.169 & 0.993 & 0.959 \\
$\varepsilon_{x, \text { un }}(\mathrm{mm} \mathrm{mrad})$ & $4.715 \times 10^{-3}$ & $14.8 \times 10^{-3}$ & $7.15 \times 10^{-3}$ \\
$\varepsilon_{y, \text { un }}(\mathrm{mm} \mathrm{mrad})$ & $4.84 \times 10^{-3}$ & $14.6 \times 10^{-3}$ & $7.22 \times 10^{-3}$ \\
\hline \hline
\end{tabular}

with respect to Case 1 ( $\mathrm{rf}$ compression off) because in the velocity bunching, the beam is injected off crest in the first section.

In the following, both for TSTEP simulations and measurements of Sec. VII, when dealing with doublet quadrupole scans, we will label "+, -" (",-+ ") scans where the quadrupole $Q_{T} 1$ is focusing (defocusing) in the horizontal plane, i.e., it is fed with positive (negative) current, while the second quadrupole $Q_{T} 3$ is defocusing (focusing) in the same plane, i.e., it is fed with negative (positive) current. Obviously in "+, -" ("-, +") scans the first quadrupole $Q_{T} 1$ is defocusing (focusing) while the $Q_{T} 3$ focusing (defocusing) in the vertical plane.

\section{A. Emittance variation induced in quadrupole scan}

As shown before, the emittance at the exit of a quadrupole is different from the one at the entrance because of chromatic effects resulting in an emittance variation proportional to the quadrupole field gradient. Figure 6 shows the emittance at the exit of a quadrupole compared to the analytical expectations of Eq. (16) (no correlations) and Eq. (20) (correlation included) for the three configurations of Table I; the gradient range is the typical range usual in SPARC operation.

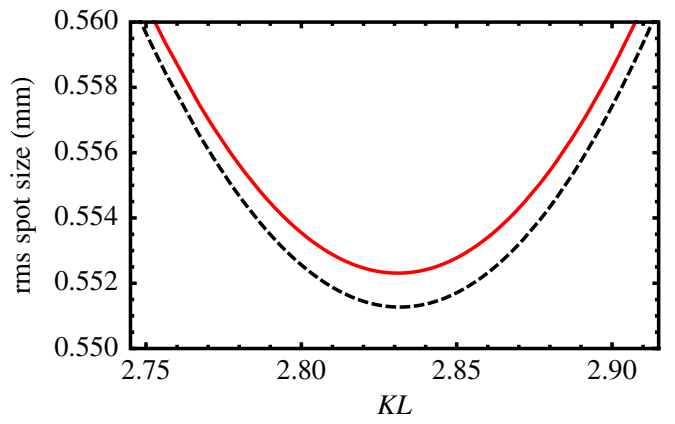

The emittance variation induced by chromatic effects in the quadrupole is negligible in Case 1 where the energy spread is low. Concerning Cases 2 and 3 (both with an energy spread around $1 \%$ but with a different spot size), the influence of the quadrupole on the emittance itself is much more remarkable with respect to Case 3 due to the dependence of $\varepsilon_{c}^{2}$ on the fourth power of the spot size [Eq. (16)]. The simulations agree perfectly with Eq. (20), accounting the computed correlations between transverse coordinates and energy induced by rf, space charge, and solenoids during the beam transport in the linac. Such correlations do affect the chromatic contribution to the emittance evidencing also a slightly emittance compensation for some quadrupole gradients.

On the contrary, Eq. (16) gives always a symmetric emittance growth with respect to the zero quadrupole gradient value. However, the approximate Eq. (16) contains only measurable quantities and therefore it can be used in practice to evaluate approximately the chromatic induced emittance, since the values of correlation terms are usually unknown.

Figures 7 and 8 compare the horizontal and vertical emittance at the exit of a single and doublet quadrupole transport for Case 2 and Case 3: as expected, the emittance variation is bigger when two quadrupoles are used. As discussed in Sec. V, the doublet quadrupole scan showed an asymmetry in the two planes: the induced chromatic emittance is larger in the plane in which the first quadrupole is defocusing. Such an effect is particularly relevant in Case 2 where the high energy spread is coupled to a large spot size. It is worthwhile to observe that also for the doublet quadrupole scan a partial compensation of emittance appears because of correlations between transverse coordinates and energy; such correlations are not accounted in Eq. (31).

\section{B. Quadrupole-scan virtual emittance measurements}

Tables II, III, and IV summarize the results of virtual measurements for the three cases of Table I: the reference

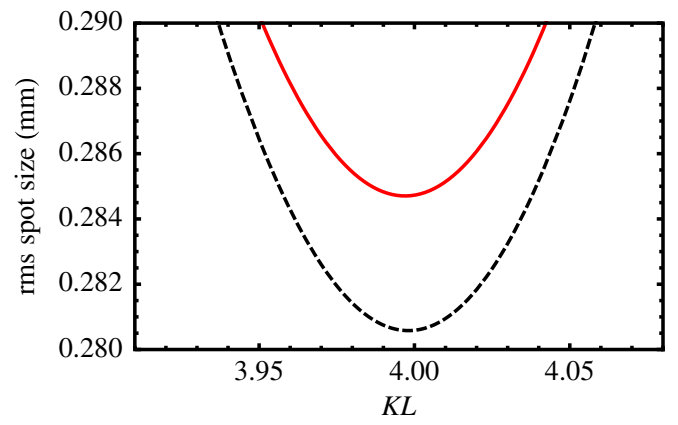

FIG. 5. Variation of the rms spot size on the measurement screen for a doublet quadrupole scan with (red solid line) and without (black dashed line) energy spread, being the first quadrupole focusing (defocusing) in the left (right) plot. $\left(\sigma_{x}=2 \mathrm{~mm}, \sigma_{x^{\prime}}=\right.$ $100 \mu \mathrm{rad}, \sigma_{x^{\prime} x}=\sigma_{x x^{\prime}}=0, L=4.5 \mathrm{~m}, \sigma_{\gamma}=1 \%$ ). 

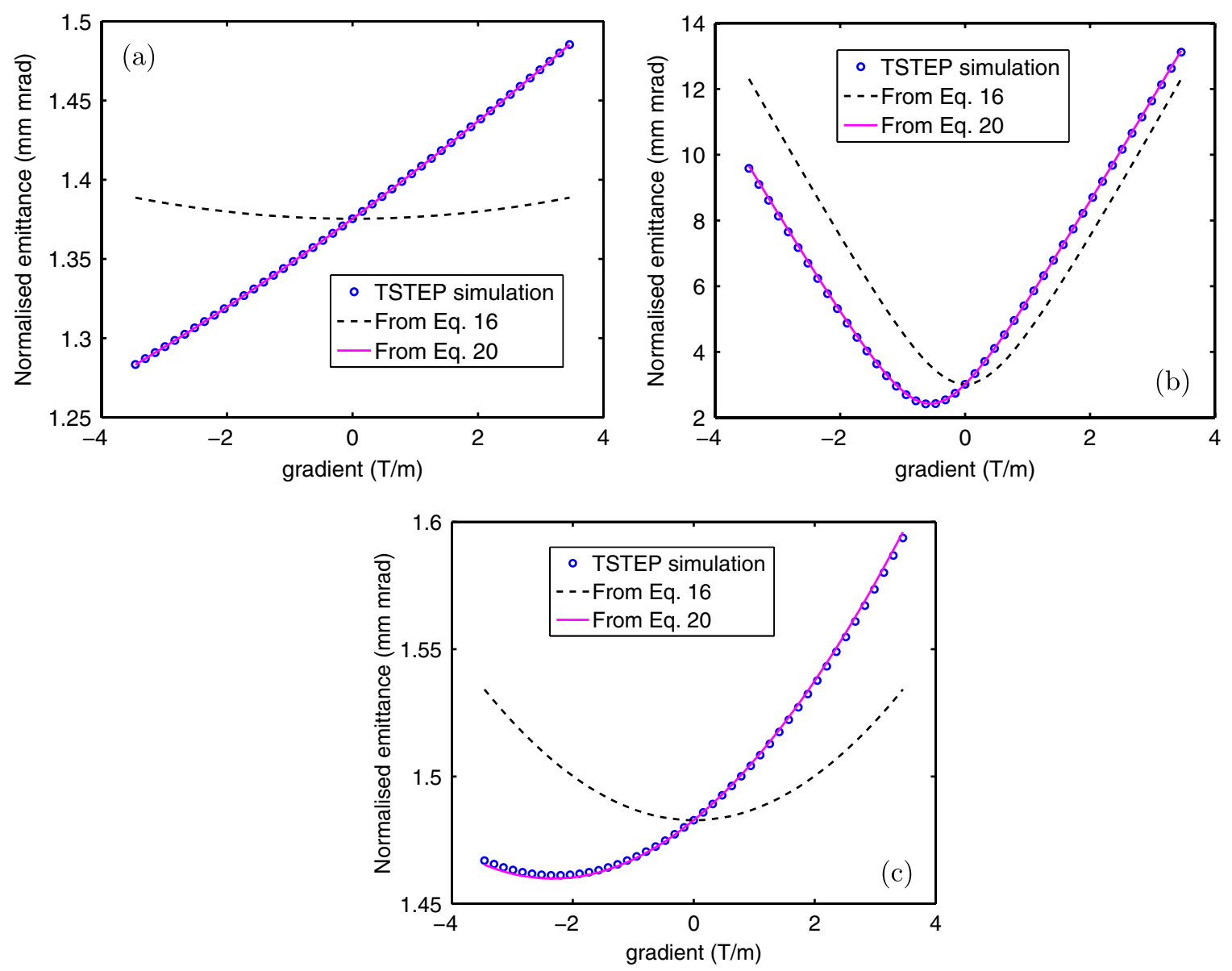

FIG. 6. Horizontal emittance as a function of gradient at the quadrupole exit for the three cases of Table I. The blue circles are the simulation results, while the black dashed line is the analytical curve with no correlations included [i.e. Eq. (16)] and the red solid line accounts for correlation [i.e. Eq. (20)]; (a) Case 1, (b) Case 2, (c) Case 3.

values in the first column are the beam emittance computed at the linac exit (screen $F_{0}$ in Fig. 1), while the values in the other columns are the values retrieved for different measurement configurations.

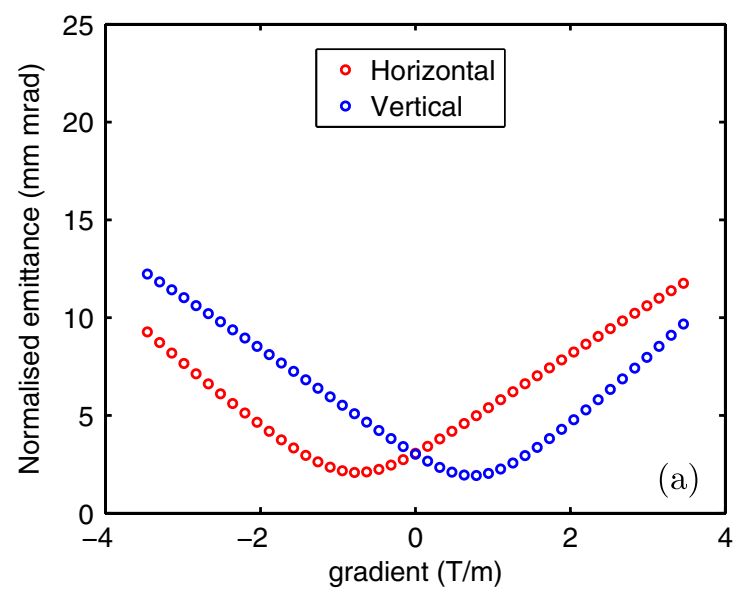

For a low energy spread beam (Case 1), the agreement between the single quadrupole scan result and with the reference value is within $0.4 \%$. In the two-quadrupoles scan, the measured value agrees within $0.5 \%$ only in the

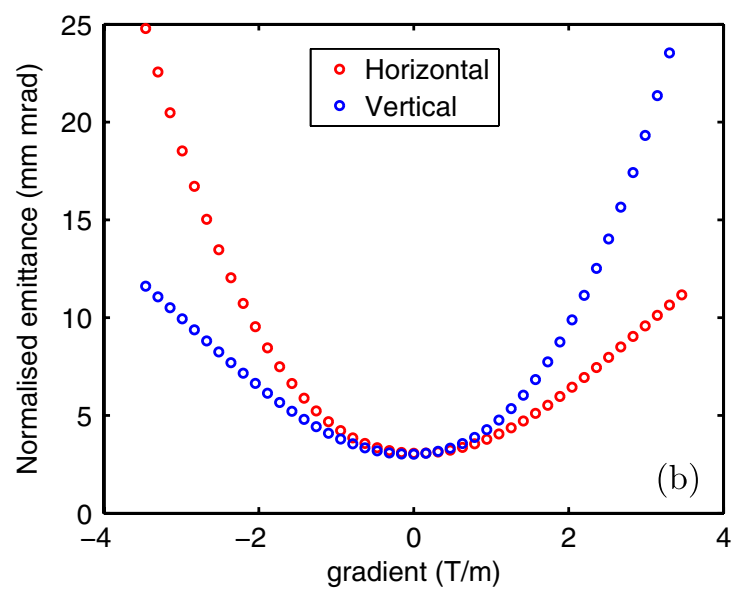

FIG. 7. Case 2: normalized horizontal and vertical emittance at the exit of the second quadrupole as a function of quadrupole gradient in a single (a) and doublet quadrupole (b) scan. In the doubler scan, the horizontal axis refers to the gradient in the first quadrupole where positive gradients are focusing in the horizontal plane. 

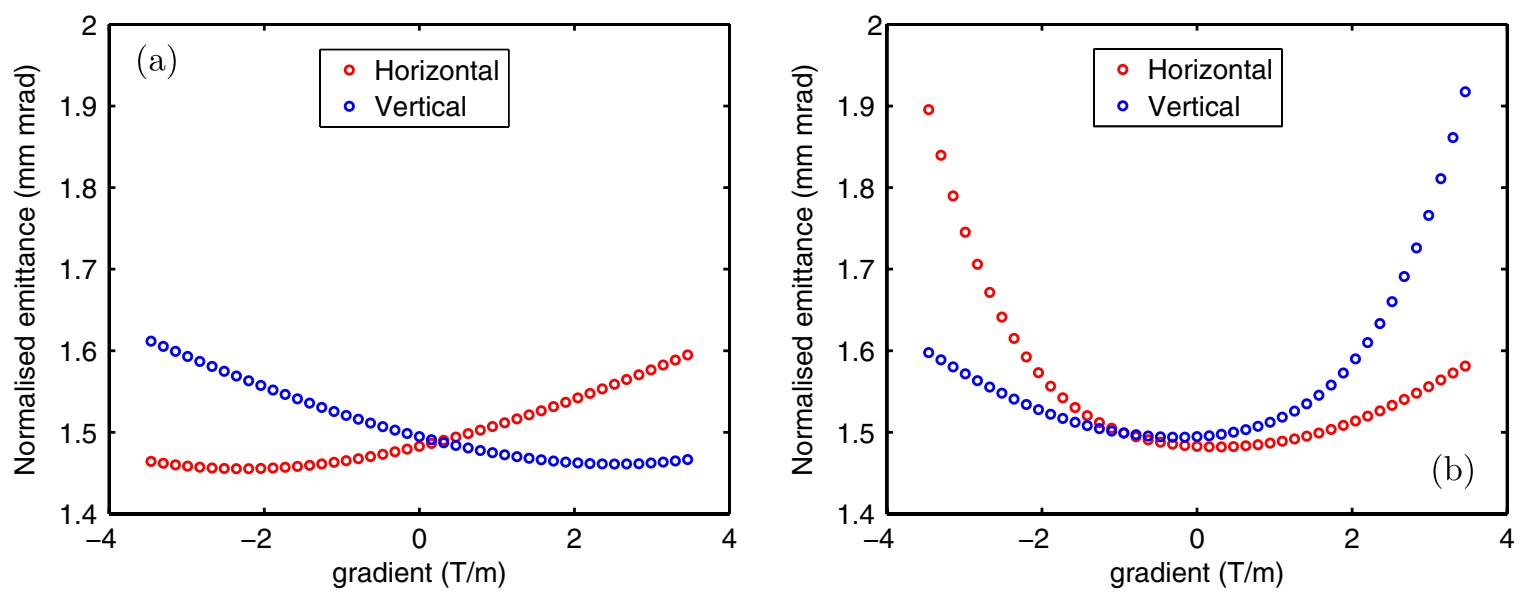

FIG. 8. Case 3: normalized horizontal and vertical emittance at the exit of the second quadrupole as a function of quadrupole gradient in a single (a) and doublet quadrupole (b) scan. In the doubler scan, the horizontal axis refers to the gradient in the first quadrupole where positive gradients are focusing in the horizontal plane.

plane in which the first quadrupole $\left(Q_{T} 1\right)$ is focusing (i.e. horizontal in "+- " configuration and vertical in the "-+" one); otherwise the emittance is overestimated (Table II).

The greatest error in the emittance estimation occurs when the beam has large energy spread and a large spot size at the first quadrupole entrance, as shown in Table III. This situation corresponds to SPARC operation in VB mode when the solenoid around the first accelerating section is switched off. In the single quadrupole scan a disagreement as big as $30 \%$ occurs while in the twoquadrupoles configuration the disagreement is within $40 \%$ only in the plane where the first quadrupole is focusing; otherwise it increases dramatically up to $200 \%$, completely spoiling the measurement.

TABLE II. Virtual emittance measurement (TSTEP simulation) for different quadrupole scans compared to the expected value (Case 1 of Table I).

\begin{tabular}{lcccc}
\hline \hline & $\begin{array}{c}\text { Reference } \\
\text { value }\end{array}$ & $\begin{array}{c}\text { Single } \\
\text { quad }\end{array}$ & $\begin{array}{c}\text { Two quads } \\
(+,-)\end{array}$ & $\begin{array}{c}\text { Two quads } \\
(-,+)\end{array}$ \\
\hline$\varepsilon_{n, x}(\mathrm{~mm} \mathrm{mrad})$ & 1.375 & 1.372 & 1.380 & 1.535 \\
$\varepsilon_{n, y}(\mathrm{~mm} \mathrm{mrad})$ & 1.413 & 1.419 & 1.590 & 1.420 \\
\hline \hline
\end{tabular}

TABLE III. Virtual emittance measurement (TSTEP simulation) for different quadrupole scans compared to the expected value (Case 2 of Table I).

\begin{tabular}{lcccc}
\hline \hline & $\begin{array}{c}\text { Reference } \\
\text { value }\end{array}$ & $\begin{array}{c}\text { Single } \\
\text { quad }\end{array}$ & $\begin{array}{c}\text { Two quads } \\
(+,-)\end{array}$ & $\begin{array}{c}\text { Two quads } \\
(-,+)\end{array}$ \\
\hline$\varepsilon_{n, x}(\mathrm{~mm} \mathrm{mrad})$ & 3.07 & 4.32 & 4.78 & 8.76 \\
$\varepsilon_{n, y}(\mathrm{~mm} \mathrm{mrad})$ & 3.02 & 4.38 & 9.07 & 4.87 \\
\hline \hline
\end{tabular}

Eventually Table IV concerns a beam with large energy spread but small spot size at the linac exit, occurring in actual VB operations at SPARC in the presence of solenoid focusing on the first linac section. In this case despite the large energy spread, single quadrupole measurement agrees very well with the actual emittance (i.e. the reference value). Also for the double quadrupole scan measurement, the agreement between the measured and the actual value improves with respect to the case of Table III where the large energy spread is combined with a large spot.

\section{Effect of sampling of the quadrupole gradient}

It is well known that, also in the absence of chromatic effects, the accuracy of the emittance measurement depends on the sampling rate of the quadrupole gradient while performing the spot size scan. In order to clarify this aspect for single and doublet quadrupole scan, we used again a virtual measurement.

Different simulated quad-scan measurements have been done by varying the quadrupole gradient step on a fictitious monochromatic beam, obtained by assigning to all macroparticles exiting from the linac (for the Case 2 of Table I) an energy value equal to the beam average energy while keeping the transverse coordinates

TABLE IV. Virtual emittance measurement (TSTEP simulation) for different quadrupole scans compared to the expected value (Case 3 of Table I).

\begin{tabular}{lcccc}
\hline \hline & $\begin{array}{c}\text { Reference } \\
\text { value }\end{array}$ & $\begin{array}{c}\text { Single } \\
\text { quad }\end{array}$ & $\begin{array}{c}\text { Two quads } \\
(+,-)\end{array}$ & $\begin{array}{c}\text { Two quads } \\
(-,+)\end{array}$ \\
\hline$\varepsilon_{n, x}(\mathrm{~mm} \mathrm{mrad})$ & 1.483 & 1.483 & 1.476 & 1.540 \\
$\varepsilon_{n, y}(\mathrm{~mm} \mathrm{mrad})$ & 1.495 & 1.490 & 1.555 & 1.491 \\
\hline \hline
\end{tabular}


TABLE V. Virtual emittance measurement (TSTEP simulation) for different quadrupole scans of the same beam (parameters of Case 2 in Table I, but with $\sigma_{\gamma}=0$ ) compared to the expected value $\varepsilon_{\text {ref }}$.

\begin{tabular}{lcl}
\hline \hline & $\begin{array}{c}\left(\varepsilon-\varepsilon_{\text {ref }}\right) / \varepsilon_{\text {ref }} \\
\text { (horizontal plane) }\end{array}$ & $\begin{array}{c}\left(\varepsilon-\varepsilon_{\text {ref }}\right) / \varepsilon_{\text {ref }} \\
(\text { vertical plane) }\end{array}$ \\
\hline Single quad & $-1.6 \% /-3 \%^{\mathrm{a}}$ & $-1.6 \% /-3 \%^{\mathrm{a}}$ \\
Two quads $(+,-)$ & $-1.9 \% /-4 \%^{\mathrm{a}}$ & $+1.98 \% /+66 \%^{\mathrm{a}}$ \\
Two quads $(-,+)$ & $+1.4 \% /+57 \%^{\mathrm{a}}$ & $-1.7 \% /-5 \%^{\mathrm{a}}$ \\
\hline \hline
\end{tabular}

${ }^{\mathrm{a}}$ The results are obtained with a gradient step of $0.16 \mathrm{~T} / \mathrm{m}$, against the step of $0.08 \mathrm{~T} / \mathrm{m}$ for all the others scans $(300 \mathrm{pC}$ beam).

unmodified. In such a way chromatics effects are completely excluded.

The results for two different steps $(0.08 \mathrm{~T} / \mathrm{m}$ and $0.16 \mathrm{~T} / \mathrm{m})$ and for single and doublet quadrupole scans are reported in Table $\mathrm{V}$ where, for the same beam, the results labeled with the superscript $a$ are obtained with a gradient step double the one used in the other measurements. In both cases (i.e. in absence of energy spread) the emittance does not change in quadrupoles but a difference with respect to the reference value remains due to undersampling.

Table $\mathrm{V}$ shows that a step of $0.08 \mathrm{~T} / \mathrm{m}$ keeps the error within $2 \%$ for both quadrupole configurations, while a doubling of the step gives a sensitively larger error for the two-quadrupole scan in the plane where the first quadrupole is defocusing (the same asymmetric behavior observed for nonzero energy spread).

Figure 9 shows the plots of the typical graphical output of the SPARC quadrupole scan tool applied to the doublet quadrupole scan of Table $\mathrm{V}$ in the horizontal plane. When the gradient sampling is too high the identification of the spot size minimum is poor [Fig. 9(a)] and the reconstructed beam ellipse may not be uniquely defined [Fig. 9(b)], despite the fit curve well reproduces the spot size data. Improving the gradient sampling, the spot size minimum is better reconstructed [Fig. 9(c)] and the trace space lines (defined in Sec. II) well enclose the beam ellipse [Fig. 9(d)]. Such visualization is used on-line by SPARC operators to quickly identify the possibility of systematic errors due to spot size under sampling during emittance measurements.
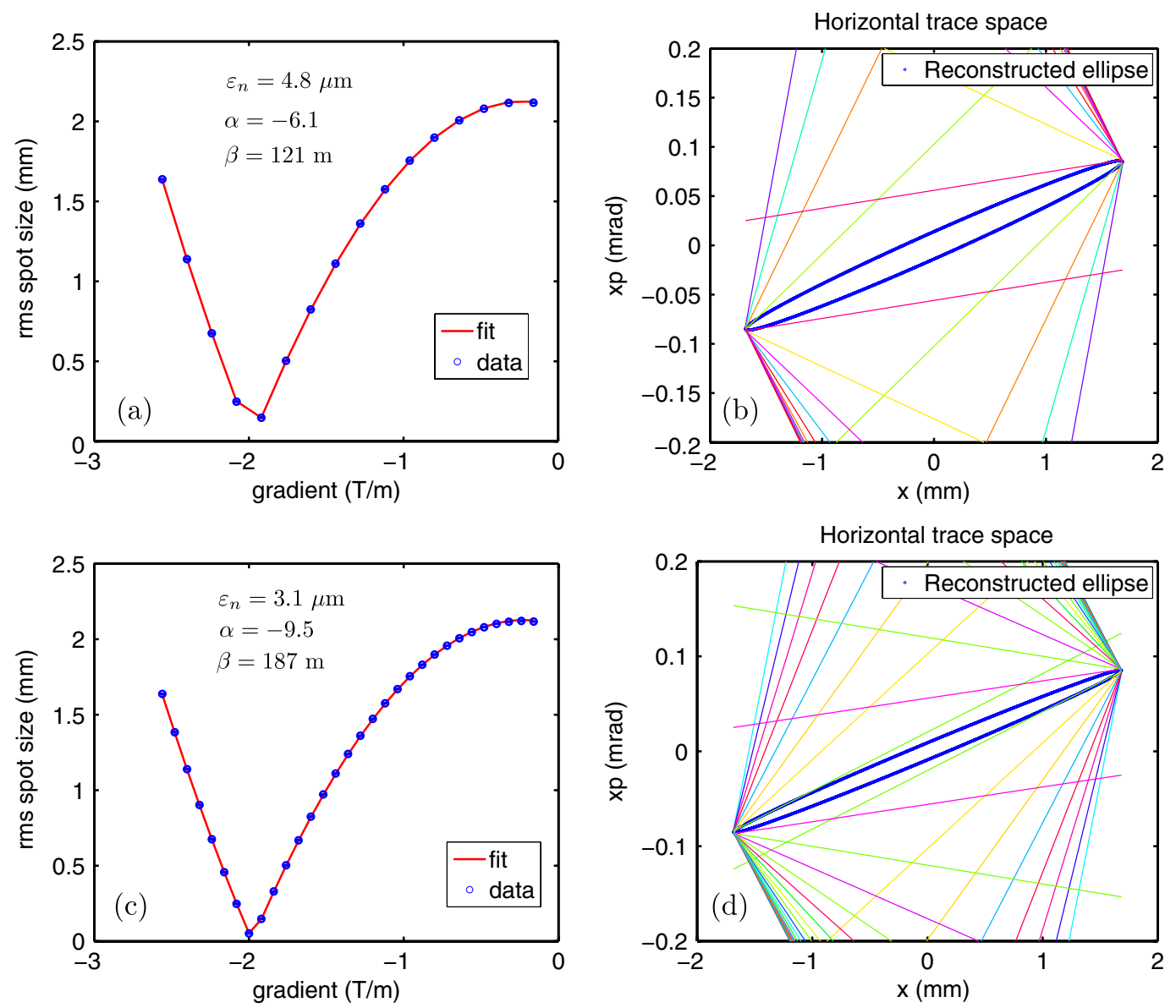

FIG. 9. Virtual emittance measurement (TSTEP simulations) for a two-quadrupole scan (" -, +" configuration). Top (bottom) plots show the spot size scan and the corresponding trace space reconstruction for a quadrupole gradient sampling step of $0.16(0.08) \mathrm{T} / \mathrm{m}$. 
TABLE VI. Emittance measurement at SPARC for low energy spread and moderate spot size at the first quadrupole entrance: $200 \mathrm{pC}, \quad \sigma_{\gamma}=0.1000(0.0040) \%, \quad \sigma_{x, F_{0}}=0.320(0.017) \mathrm{mm}$, $\sigma_{y, F_{0}}=0.3567(0.0067) \mathrm{mm}$ (analogous to Case 1 of Table I).

\begin{tabular}{lccc}
\hline \hline & $\begin{array}{c}\text { Single } \\
\text { quad }\end{array}$ & $\begin{array}{c}\text { Two quads } \\
(+,-)\end{array}$ & $\begin{array}{c}\text { Two quads } \\
(-,+)\end{array}$ \\
\hline$\varepsilon_{n, x}(\mathrm{~mm} \mathrm{mrad})$ & $1.607(0.075)$ & $1.852(0.083)$ & $2.44(0.20)$ \\
$\varepsilon_{n, y}(\mathrm{~mm} \mathrm{mrad})$ & $1.41(0.26)$ & $2.54(0.46)$ & $1.77(0.22)$ \\
\hline \hline
\end{tabular}

\section{MEASUREMENTS}

The disagreement shown by simulated emittance measurements in the previous section between single and multiquadrupoles scan has been investigated experimentally for different beam settings at SPARC. In particular, in this section we first report the experimental results for the on crest beam (i.e., maximum energy at the linac exit, no VB compression) with rms energy spread $\sigma_{\gamma}$ roughly $0.1 \%$ and measured rms spot size at $F_{0}$ of few hundreds of $\mu \mathrm{m}$; this case is analogous to Case 1 of Table I. Afterwords we focus on VB compressed beam with rms energy spread $\sigma_{\gamma}$ in the $1 \%$ range and large rms spot $(>1 \mathrm{~mm})$ at $F_{0}$; this case is analogous to Case 2 of Table I. In both measurements the beam charge was approximately $200 \mathrm{pC}$.

Table VI reports the emittance measurements (with uncertainties) with single and two-quadrupole scans for an on crest SPARC beam. The values for the two-quadrupole configuration are systematically higher than those measured in a single quadrupole scan. Furthermore, as expected from simulations of Sec. VIB, the emittance is always higher in the plane where the first quadrupole is defocusing. It means that second order effects might affect the measurement also for low energy spread and moderate spot size at the first quadrupole entrance.

On the contrary, a significant beam energy spread and a large rms spot size at the linac exit are typical of VB compressed SPARC beams when the linac focusing solenoids are not used. Figure 10 shows the same transverse trace space as reconstructed from a single quadrupole (left plot) and two-quadrupole scans ("+-" in the central plot and " $-+"$ in the right one). Estimated emittance and Twiss parameters can be strongly affected by measurement artifacts, as shown by the $\alpha, \beta, \varepsilon_{n}$ values reported on the plots. Therefore such measurements cannot be used for a safe matching on any transport beam line, as typically needed.

\section{CONCLUSIONS}

Chromatic effects on quadrupole scans for emittance measurements have been evaluated by means of approximate analytical formulas, numerical simulations, and experiments at the SPARC facility. Single and multiple quadrupole scans have been compared; the analysis has shown that, despite some practical advantages, the twoquadrupole scan measurement may introduce some errors in the emittance evaluation for beams with high energy spread and large spot size at the first quadrupole. Moreover, also for low energy spread beams an inaccurate identification of the spot size minimum due to undersampling in the quadrupole gradient scan can affect the measurement in a different way for different quadrupole configurations.

\section{ACKNOWLEDGMENTS}

This work profited from the discussion and the help of the whole SPARC team. We would like to mention also C. Biscari for her suggestions on the visualization of the measured transverse trace space which we are routinely using in the SPARC quad-scan measurements.

\section{APPENDIX: EXTENDED FORMULAS USED IN THE QUADRUPOLE-SCAN DATA ANALYSIS}

The least square method estimates for $\beta \varepsilon, \alpha \varepsilon, \gamma \varepsilon$ are given by the solution of the system written in matrix formalism as

$$
\left(\begin{array}{c}
\sum_{j=1}^{N}\left[\sigma_{j} A_{j} / u\left(\sigma_{j}^{2}\right)\right]^{2} \\
2 \sum_{j=1}^{N} A_{j} B_{j}\left[\sigma_{j} / u\left(\sigma_{j}^{2}\right)\right]^{2} \\
\sum_{j=1}^{N}\left[\sigma_{j} B_{j} / u\left(\sigma_{j}^{2}\right)\right]^{2}
\end{array}\right)=\left[T_{\ell m}\right]\left(\begin{array}{c}
\beta \varepsilon \\
-\alpha \varepsilon \\
\gamma \varepsilon
\end{array}\right),
$$
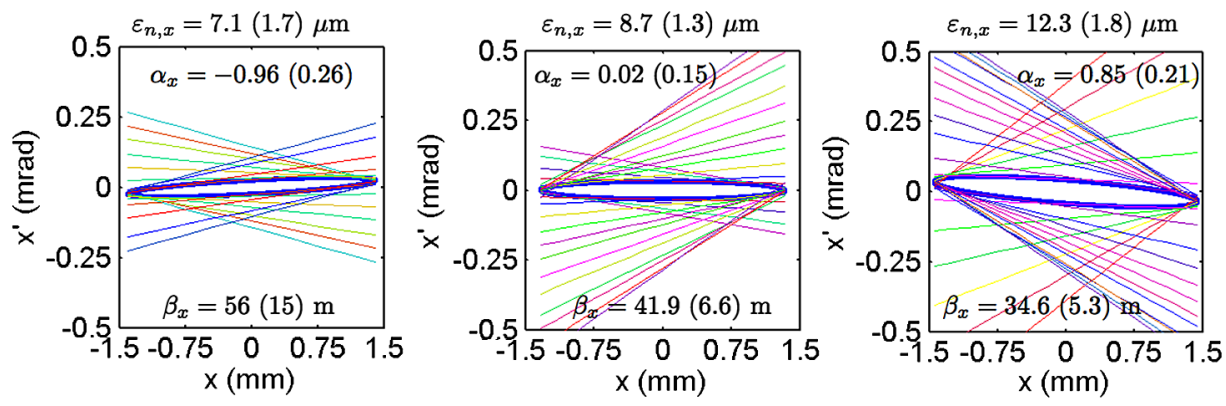

FIG. 10. Horizontal trace space for a $200 \mathrm{pC}$ VB compressed SPARC beam with $\sigma_{\gamma}=0.860(0.030) \%, \sigma_{x, F_{0}}=1.400(0.020) \mathrm{mm}$ beam (analogous to Case 1 of Table I). The same trace space is reconstructed from a single quadrupole scan (left plot) and from doublet quadrupoles "+ -" ("- +") scan in the center (right) plot. 
where

$$
\begin{aligned}
T_{11} & =\sum_{j=1}^{N} \frac{A_{j}^{4}}{u\left(\sigma_{j}^{2}\right)^{2}}, \quad T_{22}=4 \sum_{j=1}^{N}\left[\frac{A_{j} B_{j}}{u\left(\sigma_{j}^{2}\right)}\right]^{2}, \\
T_{33} & =\sum_{j=1}^{N} \frac{B_{j}^{4}}{u\left(\sigma_{j}^{2}\right)^{2}} T_{12}=T_{21}=2 \sum_{j=1}^{N} \frac{A_{j}^{3} B_{j}}{u\left(\sigma_{j}^{2}\right)^{2}}, \\
T_{13} & =T_{31}=\sum_{j=1}^{N}\left[\frac{A_{j} B_{j}}{u\left(\sigma_{j}^{2}\right)}\right]^{2}, \quad T_{23}=T_{32}=2 \sum_{j=1}^{N} \frac{A_{j} B_{j}^{3}}{u\left(\sigma_{j}^{2}\right)^{2}} .
\end{aligned}
$$

The product $\left[T_{\ell m}\right] X$, where $X$ is the 3 -vector $\beta \varepsilon, \alpha \varepsilon, \gamma \varepsilon$, is equal to the $N$ vector of expected values for the $N$ measurements, which can be considered as elements of the $N$-vector $Y$, i.e., in short notations Eq. (5) becomes

$$
Y=\left[T_{\ell m}\right] X
$$

and with some matrix algebra the vector of Twiss parameters, $X$, can be obtained:

$$
X=\left(\left[T_{\ell m}\right]^{T}\left[T_{\ell m}\right]\right)^{-1}\left[T_{\ell m}\right]^{T} Y .
$$

The error on the fit parameters is given by the covariance matrix defined as

$$
V=\left(\begin{array}{ccc}
\sigma_{\beta \varepsilon}^{2} & \operatorname{cov}(\beta \varepsilon, \alpha \varepsilon) & \operatorname{cov}(\beta \varepsilon, \gamma \varepsilon) \\
\operatorname{cov}(\alpha \varepsilon, \beta \varepsilon) & \sigma_{\alpha \varepsilon}^{2} & \operatorname{cov}(\alpha \varepsilon, \gamma \varepsilon) \\
\operatorname{cov}(\gamma \varepsilon, \beta \varepsilon) & \operatorname{cov}(\gamma \varepsilon, \alpha \varepsilon) & \sigma_{\gamma \varepsilon}^{2}
\end{array}\right)
$$

whose elements are equal to $\left[T_{\ell m}\right]$ elements:

$$
\begin{gathered}
\left(V^{-1}\right)_{11}=\sum_{j=1}^{N} \frac{A_{j}^{4}}{u\left(\sigma_{j}^{2}\right)^{2}}, \quad\left(V^{-1}\right)_{22}=4 \sum_{j=1}^{N}\left[\frac{A_{j} B_{j}}{u\left(\sigma_{j}^{2}\right)}\right]^{2}, \\
\left(V^{-1}\right)_{33}=2 \sum_{j=1}^{N} \frac{B_{j}^{4}}{u\left(\sigma_{j}^{2}\right)^{2}} \\
\left(V^{-1}\right)_{12}=\left(V^{-1}\right)_{21}=2 \sum_{j=1}^{N} \frac{A_{j}^{3} B_{j}}{u\left(\sigma_{j}^{2}\right)^{2}} \\
\left(V^{-1}\right)_{13}=\left(V^{-1}\right)_{31}=\sum_{j=1}^{N}\left[\frac{A_{j} B_{j}}{u\left(\sigma_{j}^{2}\right)}\right]^{2} \\
\left(V^{-1}\right)_{23}=\left(V^{-1}\right)_{32}=2 \sum_{j=1}^{N} \frac{A_{j} B_{j}^{3}}{u\left(\sigma_{j}^{2}\right)^{2}}
\end{gathered}
$$

The error on the Twiss parameters can then be retrieved from error propagation, whose extended expressions are

$$
\begin{aligned}
u^{2}(\beta)=\sigma_{\beta}^{2}= & \left(\frac{\partial \beta}{\partial a_{1}}\right)^{2} \sigma_{a_{1}}^{2}+\left(\frac{\partial \beta}{\partial a_{2}}\right)^{2} \sigma_{a_{2}}^{2}+\left(\frac{\partial \beta}{\partial a_{3}}\right)^{2} \sigma_{a_{3}}^{2}+2\left(\frac{\partial \beta}{\partial a_{1}}\right)\left(\frac{\partial \beta}{\partial a_{2}}\right) \operatorname{cov}\left(a_{1}, a_{2}\right)+2\left(\frac{\partial \beta}{\partial a_{1}}\right)\left(\frac{\partial \beta}{\partial a_{3}}\right) \operatorname{cov}\left(a_{1}, a_{3}\right) \\
& +2\left(\frac{\partial \beta}{\partial a_{2}}\right)\left(\frac{\partial \beta}{\partial a_{3}}\right) \operatorname{cov}\left(a_{2}, a_{3}\right),
\end{aligned}
$$

$$
\begin{aligned}
u^{2}(\alpha)=\sigma_{\alpha}^{2}= & \left(\frac{\partial \alpha}{\partial a_{1}}\right)^{2} \sigma_{a_{1}}^{2}+\left(\frac{\partial \alpha}{\partial a_{2}}\right)^{2} \sigma_{a_{2}}^{2}+\left(\frac{\partial \alpha}{\partial a_{3}}\right)^{2} \sigma_{a_{3}}^{2}+2\left(\frac{\partial \alpha}{\partial a_{1}}\right)\left(\frac{\partial \alpha}{\partial a_{2}}\right) \operatorname{cov}\left(a_{1}, a_{2}\right)+2\left(\frac{\partial \alpha}{\partial a_{1}}\right)\left(\frac{\partial \alpha}{\partial a_{3}}\right) \operatorname{cov}\left(a_{1}, a_{3}\right) \\
& +2\left(\frac{\partial \alpha}{\partial a_{2}}\right)\left(\frac{\partial \alpha}{\partial a_{3}}\right) \operatorname{cov}\left(a_{2}, a_{3}\right)
\end{aligned}
$$

$$
\begin{aligned}
u^{2}(\varepsilon)=\sigma_{\varepsilon}^{2}= & \left(\frac{\partial \varepsilon}{\partial a_{1}}\right)^{2} \sigma_{a_{1}}^{2}+\left(\frac{\partial \varepsilon}{\partial a_{2}}\right)^{2} \sigma_{a_{2}}^{2}+\left(\frac{\partial \varepsilon}{\partial a_{3}}\right)^{2} \sigma_{a_{3}}^{2}+2\left(\frac{\partial \varepsilon}{\partial a_{1}}\right)\left(\frac{\partial \varepsilon}{\partial a_{2}}\right) \operatorname{cov}\left(a_{1}, a_{2}\right)+2\left(\frac{\partial \varepsilon}{\partial a_{1}}\right)\left(\frac{\partial \varepsilon}{\partial a_{3}}\right) \operatorname{cov}\left(a_{1}, a_{3}\right) \\
& +2\left(\frac{\partial \varepsilon}{\partial a_{2}}\right)\left(\frac{\partial \varepsilon}{\partial a_{3}}\right) \operatorname{cov}\left(a_{2}, a_{3}\right) .
\end{aligned}
$$

[1] M. Minty and F. Zimmermann, Measurement and Control of Charged Particle Beams (Springer, New York, 2003), p. 99.

[2] F. Lohl, S. Schreiber, M. Castellano, G. Di Pirro, L. Catani, A. Cianchi, and K. Honkavaara, Phys. Rev. ST Accel. Beams 9, 092802 (2006).

[3] R. Spesyvtsev, TESLA-FEL Report No. 2009-09, 2009.

[4] S. G. Anderson, J. B. Rosenzweig, G. P. LeSage, and J. K. Crane, Phys. Rev. ST Accel. Beams 5, 014201 (2002).

[5] C. Limborg, Report No. SLAC-PUB-10733, 2003.

[6] M. Ferrario et al., Phys. Rev. Lett. 104, 054801 (2010).
[7] M. Boscolo, M. Ferrario, I. Boscolo, F. Castelli, and S. Cialdi, Nucl. Instrum. Methods Phys. Res., Sect. A 577, 409 (2007).

[8] M. Migliorati et al., arXiv:1112.3971v1.

[9] P. Antici et al., J. Appl. Phys. 112, 044902 (2012).

[10] D. Alesini et al., Technical Design Report for the SPARC Advanced Photo-Injector, edited by L. Palumbo and J. B. Rosenzweig (2004) [http://www.lnf.infn.it/acceleratori/ sparc/TDR/SPARC_TDR.pdf].

[11] L. Serafini and M. Ferrario, AIP Conf. Proc. 581, 87 (2001).

[12] D. Filippetto et al., Phys. Rev. ST Accel. Beams 14, 092804 (2011). 
[13] M. Ferrario et al., Nucl. Instrum. Methods Phys. Res., Sect. A 637, S43 (2011).

[14] A. Mostacci et al., in Proceedings of IPAC2011, San Sebastián, Spain, 2011 (EPS-AG, Spain, 2011), THYB01.

[15] L. Giannessi et al., Phys. Rev. Lett. 106, 144801 (2011)

[16] The Linear Two-Mile Accelerator, edited by R. B. Neal (W.A. Benjamin, Inc., New York, 1968).

[17] E. Chiadroni et al., in Proceedings of the 9th European Workshop on Beam Diagnostics and Instrumentation for Particle Accelerators, Basel, Switzerland, 2009 (PSI, Zurich, 2009), TUPD44.

[18] X. J. Wang, T. Srinivasan-Rao, K. Batchelor, M. Babzien, I. Ben-Zvi, R. Malone, I. Pogorelsky, X. Qiu, J. Sheehan, and J. Skaritka, in Proceedings of the Particle Accelerator Conference, Dallas, TX, 1995 (IEEE, New York, 1995), MPE11.
[19] J. Buon, CERN Report No. 94-01, Vol. I, p. 89.

[20] K. Floettmann, Phys. Rev. ST Accel. Beams 6, 034202 (2003).

[21] H. Wiedemann, Particle Accelerator Physics (Springer, New York, 2007).

[22] V. Blobel, Least Square Methods, Formulae and Methods in Experimental Data Evaluation, edited by R. K. Bock et al. (European Physical Society, 1983), Vol. 3.

[23] K. R. Crandall and D. P. Rusthoi, Los Alamos National Laboratory Report No. LA-UR-97-886.

[24] The thin lens approximation is used here to analytically retrieve the formula which accounts for chromatic effects in quadrupoles. On the other hand, the SPARC emittance data analysis assumes thick lenses.

[25] TSTEP is a derivative of PARMELA code; L. M. Young, Los Alamos National Laboratory Report No. LA-UR-961835. 